\title{
Acute myocardial infarction, intraventricular thrombus and risk of systemic embolism
}

\author{
Stepan Hudec ${ }^{\mathrm{a}}$, Martin Hutyra ${ }^{\mathrm{a}}$, Jan Preceka, Jan Latal ${ }^{\mathrm{a}}$, Radomir Nykla ${ }^{\mathrm{a}}$, Miloslav Spacek ${ }^{\mathrm{a}}$, Martin Sluka ${ }^{\mathrm{a}}$, Daniel Sanak ${ }^{\mathrm{b}}$, \\ Zbynek Tudos', Karel Navratild, Ludek Pavlua ${ }^{a}$, Milos Taborsky ${ }^{a}$
}

The development of left ventricular thrombus (LVT) is a well-known and serious complication of acute myocardial infarction (AMI) due to the risk of systemic arterial embolism (SE), which is variable in its clinical picture and has potentially serious consequences depending on the extent of target organ damage. SE results in an increase in mortality and morbidity in these patients. LVT is one of the main causes of the development of ischaemic cardio-embolic cardiovascular events (CVE) after MI and the determination of the source of cardiac embolus is crucial for the initiation of adequate anticoagulant therapy in secondary prevention. Echocardiography holds an irreplaceable place in the diagnosis of LVT, contrast enhancement provides higher sensitivity. The gold standard for LVT diagnosis is cardiac magnetic resonance imaging, but it is not suitable as a basic screening test. In patients with already diagnosed LVT, it is necessary to adjust antithrombotic therapy by starting warfarin anticoagulation for at least 6 months with the need for echocardiographic follow-up to detect thrombotic residues. The effect of prophylactic administration of warfarin in high-risk patients after anterior AMI does not outweigh the risk of severe bleeding complications and does not result in a decrease in mortality and morbidity. At the present time, there is not enough evidence to use direct oral anticoagulants in this indication.

Key words: left ventricular thrombus, ST segment elevation myocardial infarction, systemic arterial embolism, stroke, therapy

Received: June 28, 2019; Revised: December 5, 2019; Accepted: January 3, 2020; Available online: January 31, 2020

https://doi.org/10.5507/bp.2020.001

(c) 2020 The Authors; https://creativecommons.org/licenses/by/4.0/

${ }^{a}$ Department of Internal Medicine I - Cardiology, University Hospital Olomouc and Faculty of Medicine and Dentistry, Palacky University, Olomouc, Czech Republic

${ }^{b}$ Comprehensive Stroke Center, Department of Neurology, University Hospital and Faculty of Medicine and Dentistry, Palacky University, Olomouc, Czech Republic

'Department of Radiology, University Hospital and Faculty of Medicine and Dentistry, Palacky University, Olomouc, Czech Republic

${ }^{d}$ Department of of Internal Medicine, Military Hospital, Olomouc, Czech Republic

Corresponding author: Jan Precek, e-mail:jan.precek@seznam.cz

\section{INTRODUCTION}

The development of left ventricular thrombus (LVT) is a well-known and serious complication of acute myocardial infarction (MI) due to the risk of systemic arterial embolism (SE). It presents with a variable clinical picture and has potentially serious consequences depending on the extent of target organ damage, most commonly the brain. Cardiovascular mortality decreased after the introduction of percutaneous coronary intervention (PCI) as the dominant reperfusion strategy for the treatment of patients with acute MI, which is superior to thrombolysis $^{1-4}$. However, LVT continues to significantly affect patient mortality and morbidity due to SE (ref..$\left.^{5,6}\right)$. The main risk factors for LVT development are the duration of myocardial ischaemia, MI size and the resulting LV kinetics disorder ${ }^{6}$. The incidence of LVT after MI decreased significantly with the introduction of pharmacological reperfusion therapy. Further reductions in LVT incidence after MI can be expected in the modern era of routine
PCI for early myocardial reperfusion within MI therapy. However, such a decrease has not been reliably demonstrated compared to thrombolysis, and LVT remains a feared complication following a prior acute ST segment elevation myocardial infarction (STEMI), particularly in the anterior wall ${ }^{7}$.

The aim of this review article is to provide comprehensive information on the incidence of LVT in the era of primary PCI, on the diagnostic methods suitable for LVT detection, the stratification of risk groups of patients predisposed to LVT development and finally on the possibilities of therapy using combined antithrombotic medication. The aim of these measures is to prevent systemic arterial embolism while minimizing bleeding episodes to achieve the maximum possible reduction in mortality and morbidity in patients with MI complicated by LVT $\left(\right.$ ref. $\left.^{8-13}\right)$. 


\section{EPIDEMIOLOGY, PATHOGENESIS, PREDISPOSITION TO LVT AND RISK OF SYSTEMIC EMBOLIZATION}

\section{Epidemiology}

Prior to the introduction of reperfusion therapy for acute MI, i.e. in the pre-thrombolytic era, the incidence of LVT in patients after STEMI was reported in a wide range of $20-40 \%$, with even up to $60 \%$ in large MI of the anterior wall ${ }^{14,15}$. This incidence decreased by introducing early reperfusion therapy for MI, both pharmacological and interventional. In the modern era of interventional therapy for acute MI, the incidence of LVT in patients after anterior $\mathrm{MI}$ is reported to be within a wide range of 4 to $15 \%$ (ref. ${ }^{16,17}$ ). The large differences in LVT incidence between the studies may be due to the different time lag of LVT detection from the onset of MI and also depends on the diagnostic sensitivity of the imaging method used to detect LVT (echocardiography, contrast-enhanced echocardiography, magnetic resonance imaging).

The term "early LVT" has been proposed when considering the time horizon since the onset of MI. It is detected during the initial hospitalization or within 7 days of the onset of MI. Late LVT is usually diagnosed within 1-3 months of MI.

In a study by Osherova et al., early thrombus was detected in $6.2 \%$ of patients (40/642 patients) and late thrombus in $15 \%$ (96/642 patients). However, only patients treated with primary PCI were included in this study; patients after thrombolysis and conservative therapy were excluded. The incidence of LVT was not significantly different when comparing patient subgroups, but mortality was lowest in patients treated with primary PCI. The above can be explained by some of the limitations of the above study, such as selection bias for patient grouping, i.e. indication of primary PCI in higher-risk patients, as well as different therapeutic strategies among groups, especially longer anticoagulation times in conservatively treated patient ${ }^{7}$. A recently published study by Maniwa et al. reported the incidence of ventricular thrombi diagnosed by echocardiography, ventriculography and magnetic resonance imaging (MRI) of the heart as $5 \%(92 / 1850)$ of patients after a recent first MI (ref. $\left.{ }^{11}\right)$. A lower incidence of LVT was described by Tiffany et al., where contrast-enhanced echocardiography (76\% of patients) was used to detect LVT. LVT was detected in $1.6 \%(28 / 1698)$ of patients, but this finding can be interpreted by the focus on the entire population of patients with STEMI in all localisations, who were examined only on the second day after MI, without the possibility of detecting thrombi formed later ${ }^{18}$. In a meta-analysis that analysed the incidence of LVT in STEMI patients in the primary PCI era, $2.7 \%$ of patients had LVT in STEMI of all localizations, but LVT occurred in $9.1 \%$ of anterior wall STEMI patients. Furthermore, an inverse relationship was observed between the size of the patient sample and the incidence of LVT. The incidence of LVT was higher the lower the number of patients evaluated in the study $(<100)$. The incidence of LVT in anterior wall STEMI was $7.5 \%$ following the exclusion of studies with a low number of evaluated and monitored patients. The time window for LVT detection was 3 to 90 days after the onset of MI. No decrease in LVT incidence was found in newer papers when comparing studies according to the date of their publication ${ }^{19}$. The incidence of LVT was $8 \%$ in studies following the first acute STEMI (all localizations) where cardiac MRI was used to detect LVT (ref. ${ }^{8,20}$ ).

\section{Pathogenesis and predisposition to LVT development}

The combination of blood stasis, endothelial damage and hypercoagulation is well known as Virchow's triad and predisposes to thrombosis development in-vivo. The Virchow's triad is originally associated with development of deep venous thrombosis, but these three predisposing factors can also be identified in ventricular thrombus formation after acute MI, particularly in transmural MI in the area of the significant coronary artery. The disorder of regional kinetics, akinesia and dyskinesia, the pres-
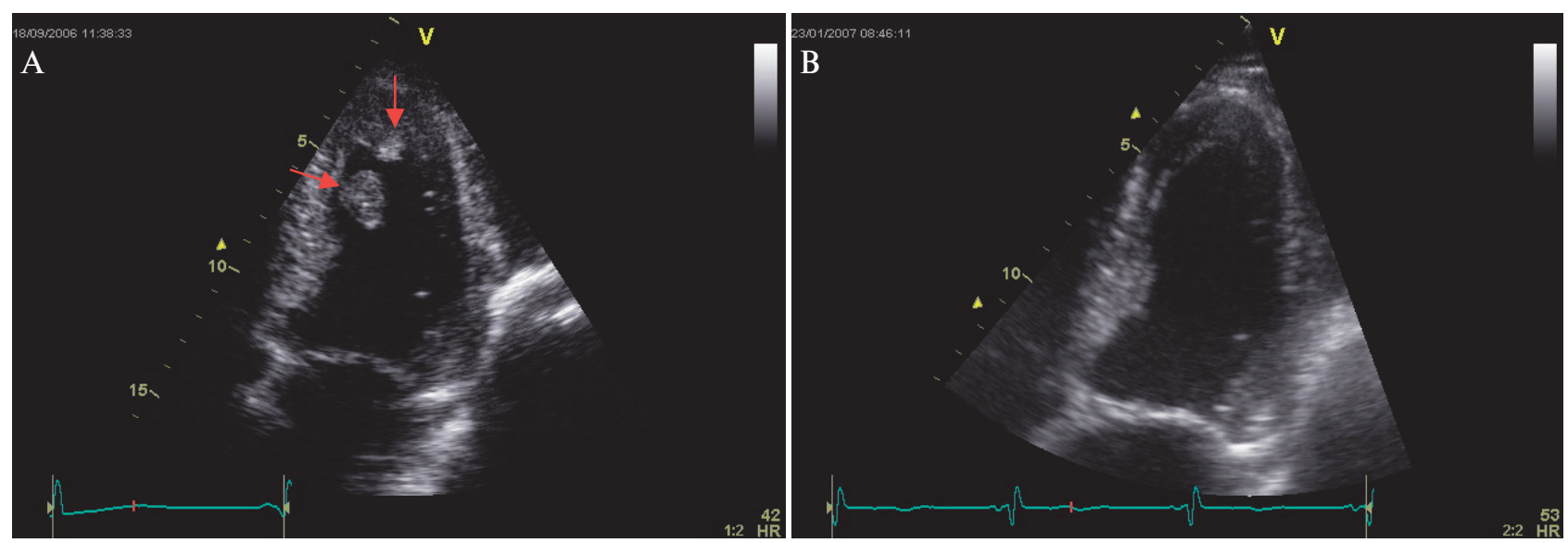

Fig. 1. Left ventricular thrombus before (A) and its complete resolution after (B) anticoagulation therapy in a patient after cardioembolic CVA immediately complicating an acute myocardial infarction of the anterior wall.

The apical four-chamber views shows a mobile thrombus apico-septally and a smaller thrombus in the apical area (arrows). Warfarin anticoagulant therapy and dual antiplatelet therapy led to the complete resolution of both thrombi after 4 months. 
ence of LV aneurysm, or mechanical complications with covered rupture and pseudoaneurysm formation of $\mathrm{LV}$ lead to blood stasis, which can sometimes be recognized echocardiographically as a spontaneous echocontrast (so called smoke phenomenon) in the LV cavity.

Prolonged myocardial ischaemia leads to endocardial damage resulting in an inflammatory response and in a hypercoagulable condition caused by the release of prothrombogenic factors such as fibrinopeptide A, von Willebrand factor (vWF), prothrombin and decreased levels of enzymes responsible for vWF removal (ADAMTS13) (ref. $^{6}$ ). LVT usually occurs in irreversibly damaged infarctions and non-viable LV segments ${ }^{21}$.

The thrombus may develop early in the first 24 hours after MI. According to Shacham et al., up to $61 \%$ of all early thrombi are formed during this time and with $90 \%$ of thrombi are being formed within 2 weeks of MI (ref. ${ }^{16}$ ). However, it should be noted that the so-called late LVT may have slightly different pathogenesis from that of early thrombi, potentially mainly depending on persistent systolic dysfunction of LV, however specific data is not available. In a control contrast-enhanced cardiac MRI study in the 4th month after STEMI, LVT was detected in $6.2 \%$ of patients (12/194 patients), while 8.8\% (17/194 patients) had early thrombi ${ }^{20}$. A spontaneous or anticoagulantinduced thrombus resolution is more common in apical akinesia, but worse in apical aneurysm or dyskinesia ${ }^{22}$.

Rupture of the free left ventricular wall is a mechanical complication of MI with a dramatically poor prognosis and occurs in approximately $1-6 \%$ of all MI patients. A lower overall incidence of this mechanical complication of MI can now be expected given the fact that most of this
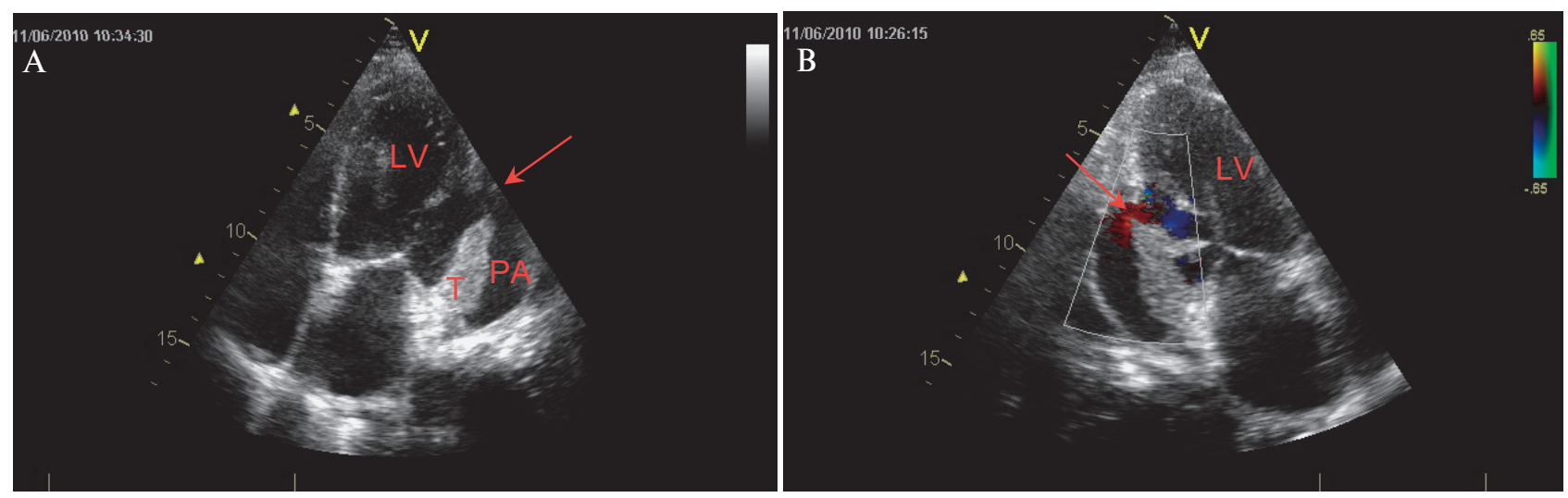

Fig. 2. A partially thrombosed pseudoaneurysm of the posterolateral left ventricular wall - echocardiography.

Apical four-chamber view (A) shows LV wall rupture at the basal and middle segment border of the anterolateral wall. The crevice marked by the arrow leads to a pseudoaneurysm with a suspected thrombus (hyperechogenic apposition on the LV basal segment wall when compared to different echogenicity of the wall of the apico-lateral segment). The apical two-chamber projection (B) shows a pulsatile flow in colour Doppler mapping (LV - left ventricle, PA - pseudoaneurysm, T - thrombus).
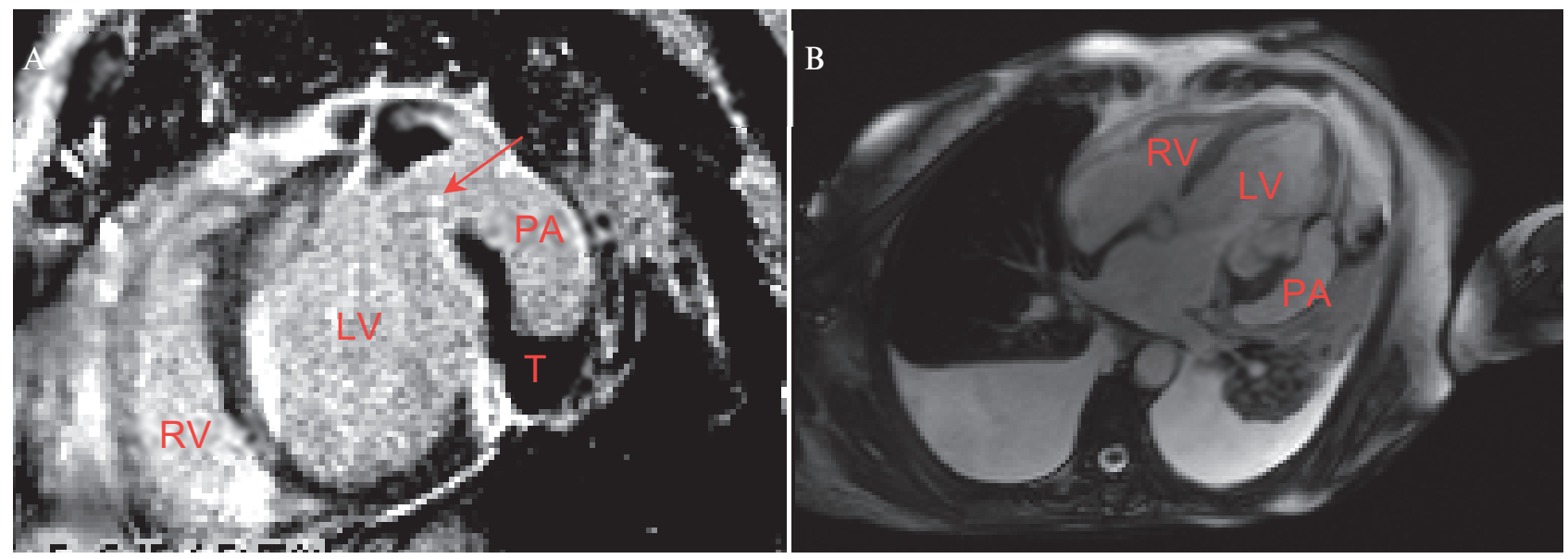

Fig. 3. A partially thrombosed pseudoaneurysm of the posterolateral wall of the left ventricle (LV) - cardiac magnetic resonance imaging.

Projections in the horizontal long axis (B) and the short axis of LV (A) in the reconstructed PSIR-TrueFisp 2D sequence using contrast medium (Gadovist) show wall discontinuity marked by an arrow - rupture of about 10mm - localized posterolateral with the development of a pseudoaneurysm (PA). Furthermore, transmural delayed post-contrast enhancement is evident throughout the posterolateral and posterobasal walls corresponding to the scar. A thrombus (T) is evident within the pseudoaneurysm. A minimal amount of fluid is present in the pericardium and fluidothorax is visible on both sides (LV - left ventricle, RV - right ventricle, PA - pseudoaneurysm, T - thrombus). 

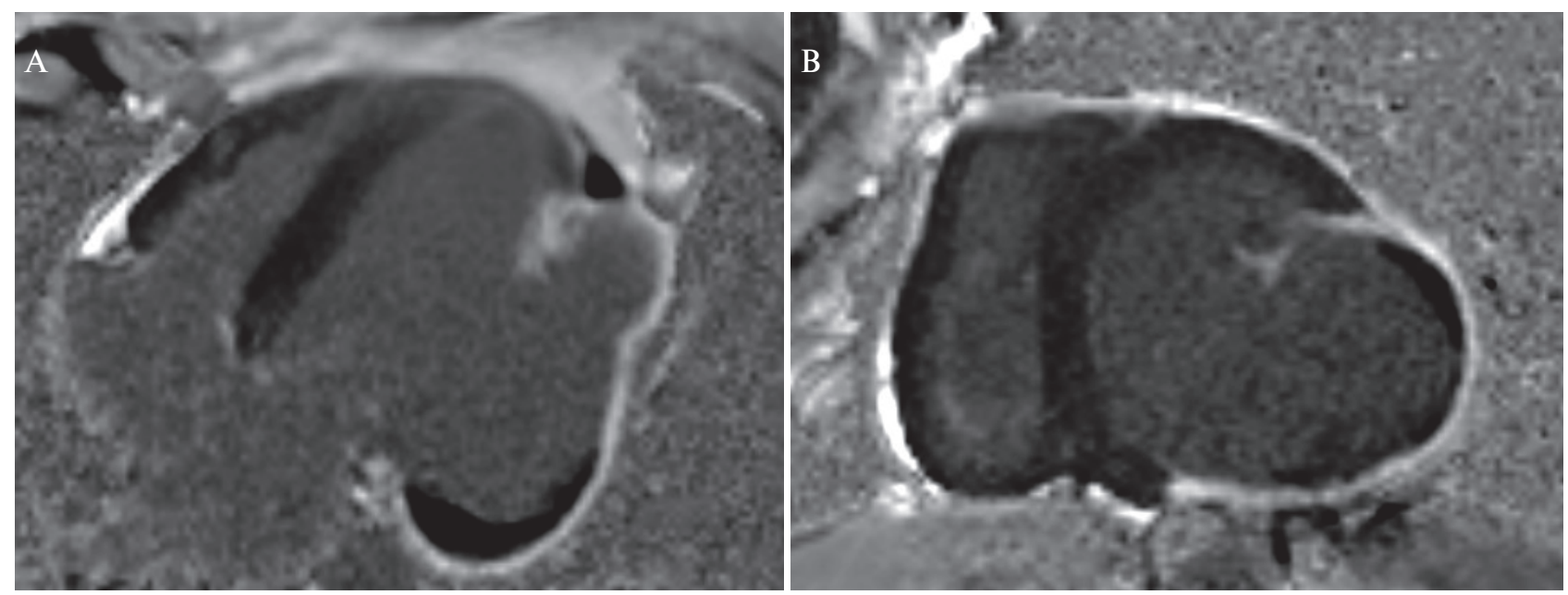

Fig. 4. Cardiac magnetic resonance imaging.

The projections in the horizontal long (A) and short axis of the LV (B) in the reconstructed PSIR-TrueFisp 2D sequence using contrast medium (Gadovist) show an aneurysmal protrusion in the posterolateral wall of the left ventricle myocardium. Furthermore, transmural delayed post-contrast enhancement is evident throughout the wall of the aneurysm, with a cranio-basally localized wall thrombus $(\mathrm{T})$ on the endocardial surface.

data comes from an era where the treatment of acute MI was predominantly pharmacological. Although the clinical picture is usually very dramatic, in some cases acute cardiac tamponade does not develop and the rupture of the heart wall is covered by surrounding pericardium, partially scarred tissue or thrombus, resulting in the formation of a pseudoaneurysm communicating with the left ventricle cavity. Unlike a true aneurysm, the wall of a pseudoaneurysm is not made up of myocardium or scar, but of surrounding tissues. This is a very rare complication that is reported in approximately $0.2 \%$ of all MI patients. The most common forms of clinical manifestation of pseudoaneurysm are signs of heart failure (36\%), chest pain $(30 \%)$ and exertional dyspnoea (20\%). Sudden death is the first symptom in about $3 \%$ of cases with only about $12 \%$ of patients being asymptomatic at the time of diagnosis. Rare clinical manifestations include cardiac arrhythmias or a stroke with systemic embolism. Most patients are admitted to hospital for 3-80 days after the previous MI. The risk of rupture of an already diagnosed pseudoaneurysm exceeds $45 \%$, while the mortality of individuals indicated for surgery does not exceed $10 \%$ compared to $48 \%$ mortality in the conservatively-managed group. Systemic embolism occurs in the cerebral circulation in $1-2.5 \%$ of patients with acute infarction, as a result of thrombus formation within 1 month, half of which occurs during the first 5 days. The risk increases depending on regional and global systolic dysfunction of the left ventricle.

The identification of independent risk factors for LVT development after MI defines better the groups of highrisk patients for the development of this complication. This can lead to a modification of the diagnostic algorithm and potentially enable early modification of antithrombotic therapy with the aim of primary prevention of clinically silent or manifest SE. It is evident from the available studies that LVT is most common in STEMIs originating in the perfusion territory of the left anterior descending artery (LAD) (ref. ${ }^{8,17,18}$ ). In the vast majority of cases, the MI affects the anterior wall ${ }^{19}$. Patients have a larger infarct size and cardiac MRI detects microvascular obstruction more frequently ${ }^{20}$. Another predictor of LVT development is LV systolic dysfunction, usually defined by a decreased LV ejection fraction (EF) below $40 \%$ and the development of LV aneurysm with typical dyskinesia of apex and adjacent parts of the LV (ref. ${ }^{5,16}$ ). However, in a large-scale echocardiographic study by Weinsaft et al., severe systolic dysfunction of the LV (LV EF <30\%) was not a necessary precondition for LVT and only one quarter of all LVT cases developed in severe LV systolic dysfunction. This may indicate that the severity of the apical kinetics disorder determined e.g. by the wall motion score in the apical segment, may be a more important predictor of LVT development after MI than the global LV systolic function evaluated based on LV EF (ref. ${ }^{8}$ ). Other predictors are longer time to successful reperfusion (time from the onset of symptoms to PCI), incomplete revascularization and post-PCI TMII flow score 1, which are generally parameters associated with the extent in size of the resulting infarct ${ }^{5,16}$. The risk of LVT can be inferred from the laboratory biomarker values of myocardial damage. Patients with anterior STEMI and creatine kinase (CK) levels higher than $4.912 \mathrm{U} / \mathrm{L}$ are at a $12 \mathrm{x}$ higher risk of developing LVT (ref. ${ }^{17}$ ). Elevation of D-dimers (cut-off $1.53 \mathrm{mg} / \mathrm{L}$ ) is associated with a higher risk of LVT and these elevated values are probably caused by prothrombogenic alterations induced by myocardial necrosis. Another predictor of LVT formation may be the SYNTAX score ( 29.5 cut-off) (ref. ${ }^{5}$. There was no association between LVT development and atherosclerosis risk factors, comorbidities including atrial fibrillation or carotid stenosis and peripheral artery disease. Furthermore, no difference was found in the $\mathrm{CHA}_{2} \mathrm{DS}_{2}$-VASc score between the LVT and LVT-free groups ${ }^{11}$.

The identification of reliable predictors of LVT development in patients after MI remains a challenge. 


\section{Risk of systemic embolism in patients with LVT}

The incidence of clinically manifest SE in LVT patients in the pre-thrombolytic era was reported to be in the range of $10 \%$ to $35 \%$; in the thrombolytic era $2-3 \%$ (ref. ${ }^{6,23}$ ). According to a meta-analysis involving 856 patients after anterior MI, the presence of LVT was associated with an approximately 5-fold higher incidence of embolic events ${ }^{24}$. However, this analysis was performed prior to the introduction of PCI as the dominant reperfusion therapy and prior to the routine use of dual antiplatelet therapy (DAPT) after MI. There is limited evidence for the risk of clinically manifest SE in patients with LVT after MI in the era of primary PCI.

In a recent study by Maniwa et al. in patients after the first MI (both non-STEMI and STEMI), clinically apparent SE occurred in $3.6 \%$ of the study population (66/1850 patients). SE occurred in $16.3 \%$ (15/92 patients) of subjects with a proven thrombus, and in $2.9 \%(51 / 1758$ patients) of subjects without the evidence of left ventricular thrombus during the follow-up period of 5.4 years. In this study, the hazard ratio (HR) for SE was also determined between the groups of patients with/without atrial fibrillation (AF) and/or LVT compared to the underlying risk group - i.e. without LVT and without AF. HR for SE was significantly higher in patients with LVT/without AF (HR 5.2) and in patients with AF/without LVT (HR 3.2). Thus, LVT development was associated with even higher $\mathrm{SE}$ risk than AF itself, with the highest $\mathrm{HR}$ in the AF/ LVT group (HR 15.0) (ref. ${ }^{11}$ ).

Contrary to that, Delewi et al. observed a relatively benign course, with no stroke reported in patients after acute STEMI complicated by LVT, regardless of the type of antithrombotic therapy (DAPT vs. triple therapy). LVT was diagnosed using contrast-enhanced cardiac MRI and patients underwent successful PCI revascularization. However, the exclusion criteria for high-risk patients (haemodynamically unstable, creatine kinase elevation, after PCI failure) were relatively extensive in this study ${ }^{20}$.

It is likely that the shape, motility and appearance of the thrombus during echocardiography or other im- aging (MRI) may affect the risk of SE, where mobile, echolucent and protruding thrombi may predispose to this complication. Organized, sessile thrombi with limited mobility have been shown to be of lower risk, but specific data is lacking ${ }^{25,26}$. It is interesting to compare the risk of $\mathrm{SE}$ in patients with chronic heart failure, where the incidence of apparent SE in clinically stable patients is only $1-3 \%$, even in patients with severe systolic dysfunction and echocardiographic evidence of intracardiac thrombus ${ }^{27-29}$.

\section{DIAGNOSIS}

The basic examination and screening method for LVT detection is echocardiography, which is performed as a standard part of the diagnostic management in patients after acute MI. Echocardiographically, a ventricular thrombus is defined as an echodense mass, sessile to a portion of the myocardium with abnormal kinetics (akinesia or dyskinesia), which is distinguishable from the adjacent myocardium and has a distinct interface between thrombus and blood in LV. It should be detected in at least two echocardiographic projections, preferably also from two acoustic windows ${ }^{30}$. LVT after acute MI is most often localized in the LV apex area and does not infiltrate the free wall of LV (as opposed to tumours), whereas up to $20 \%$ of LV thrombi in chronic heart failure may form in a non-apical position ${ }^{8}$. Morphologically, sessile or protruding thrombi can be described, with smooth or irregular contours. The appearance of the thrombus can vary and depends on its age. Early thrombi are echolucent, more mobile, and protrude more often into the LV cavity. Older thrombi usually have a smooth surface, echogenicity similar to liver tissue, and may be organized to some extent, including calcification. Thrombi usually have a higher echodensity than the adjacent myocardium and their contour is distinguishable from the contour of the endocardium.

Although echocardiography is a very useful imaging method for LVT detection, especially because of its wide availability, relatively low time demands and high mobil-
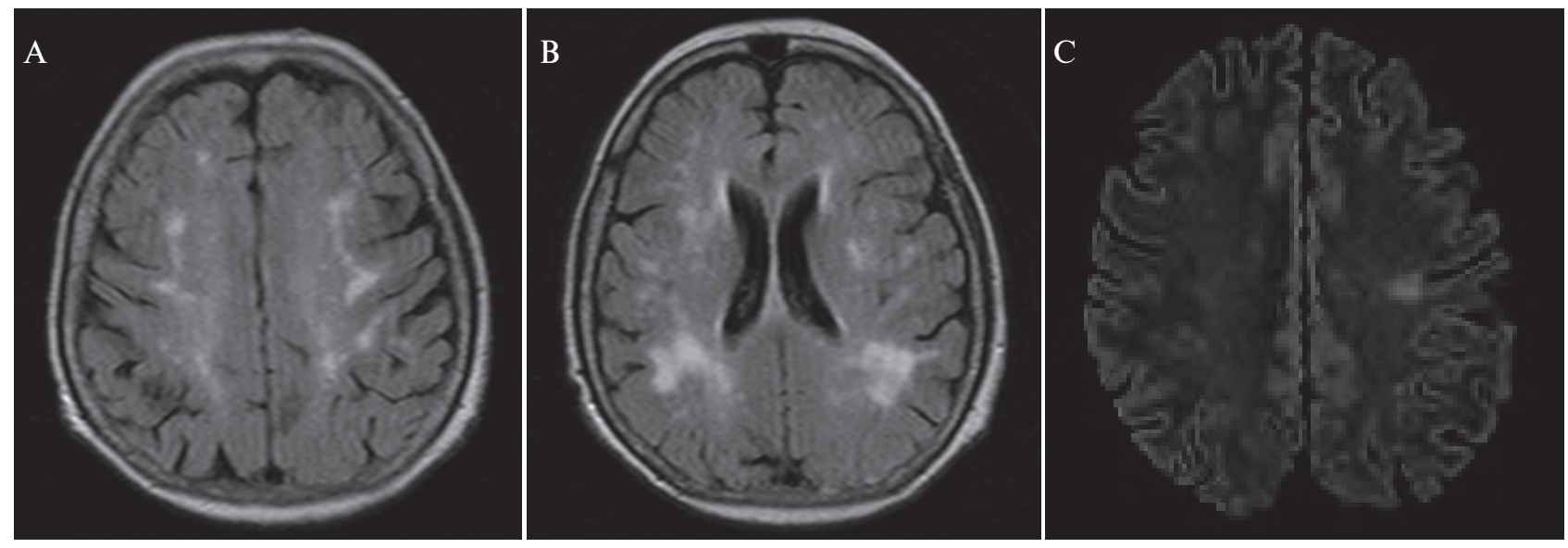

Fig. 5. Ischaemic brain lesions on magnetic resonance imaging.

A, B: Fluid attenuated inversion recovery (FLAIR) sequence. Small multiple ischaemic foci on both sides fronto-parietally. C: Diffusion-weighted imaging (DWI) sequence with ischaemic brain lesion findings consistent with the embolic lesions. 
ity of the device which allows for bed-side examinations in critically ill patients, it is limited in the detection of ventricular thrombi. Compared to the reference imaging method, i.e. the contrast-enhanced cardiac MR with delayed enhancement (DE-CMR) of myocardium phenomenon, the diagnostic accuracy of echocardiography without the use of a specific contrast agent with the possibility of left ventricular opacification in the detection of ventricular thrombi is relatively limited, with a sensitivity of $35 \%$, specificity of $98 \%$, positive and negative predictive values of $67 \%$ and $94 \%$, respectively.

Contrast-enhanced echocardiography increases the sensitivity from $33 \%$ to $61 \%$ and specificity from $98 \%$ to $99 \%\left(\right.$ ref. $\left.^{8}\right)$. The suboptimal sensitivity of echocardiography may be caused by the difficulty in differentiating a LVT from cardiac trabeculae or other muscle structures. Contrast-enhanced echocardiography helps differentiate these structures - after contrast media administration, LVT looks like a defect not filled by contrast separated from the myocardium, while spontaneous echocontrast (smoke) and muscle cardiac structures are hidden by the contrast. Concentric echoes in the apical projection area of the scanned sector may sometimes be the source of a false diagnosis of a thrombus in the LV apex - the near field artefact. Therefore, it is recommended to use the maximum possible probe frequency and decrease the depth of the examination field. Weinsaft et al. evaluated the regional LV kinetics according to a 17-segment model with a 5-point scale myocardial contractility evaluation. Due to the predilection of LVT development after MI, for the LV apex (94\% of all thrombi developed apically in this study), they focused on assessing the so-called wall motion score in this area (total of 5 segments). In apical wall motion scores of $\geq 5$ in non-contrast echocardiography or $\geq 7$ in contrast-enhanced echocardiography, a sensitivity of $100 \%$ was achieved as well as a negative predictive value and specificity of $61 \%$ and $68 \%$, respectively. In view of the high negative predictive value, up to $56 \%$ or $63 \%$ of patients could avoid further testing with DE-CMR and the determination of apical wall motion score would reduce the number of patients indicated for this examination. Thus, it would be a useful tool for the effective stratification of patients into risk groups where DE-CMR is really necessary to detect LVT. It should be noted that the determination of this score is quite dependent on the subjective assessment of the investigator. Therefore, other parameters are sought after, which would be more independent of the investigator's subjective assessment.

A promising echocardiographic method in this issue could be myocardial deformation analysis (speckletracking echocardiography). After a routinely performed $2 \mathrm{D}$ echocardiography with the acquisition of the necessary projections, it is possible to evaluate the necessary parameters using specialized software (post-processing). In this way, it is possible to determine the regional or global 2D longitudinal strain of LV (GLS), which reflects regional kinetics disorders and assesses global systolic function of LV using myocardial deformation rate (strain $-\%)$. Certain levels of myocardial strain may be associated with a higher risk of LVT development and may help to better define the group of patients indicated for DE-CMR. Determination of regional (apical) strain may be more accurate than the determination of the apical wall motion score. In myocardial deformation analysis in patients after anterior STEMI, both GLS and apical strain were approximately 2-fold lower in patients with LVT compared to patients without ${ }^{31}$.

The use of oesophageal echocardiography (TOE) in the diagnosis of LVT is problematic, namely due to a worse visibility of LV apex. Moreover, it is a semi-invasive method with worse subjective tolerability. Improved apical imaging is achieved by using a deep transgastric projection or by using a $3 \mathrm{D}$ probe, which is not available in all centres $^{26,32}$. The reported TOE sensitivity and specificity are $40 \%$ and $96 \%$, respectively ${ }^{33}$.

Cardiac computed tomography (CT) has comparable sensitivity and specificity to B-mode transthoracic echocardiography, but is associated with increased exposure to ionizing radiation and intravenous contrast (CT contrast) with the potential risk of nephrotoxicity or allergic reactions. Tissue differentiation is also worse with CT when compared to CMR, therefore, the role of CT is limited in LVT detection and its use is not widespread for this indication ${ }^{6}$. The gold standard and reference method in LVT detection is DE-CMR, which defines LVT in the T1 sequence as a hyposignal (dark, avascular) mass surrounded by hypersignal structures such as intracavitary blood and infarction focus with late gadolinium enhancement. LVT must be distinguished from microvascular obstruction in a heart attack, which is also seen as a hyposignal mass (dark area) (ref. ${ }^{34}$ ). In a study by Srichaie et al., the sensitivity and specificity of DE-CMR for the diagnosis of LVT was compared with TTE and TOE in 361 patients with coronary artery disease prior to cardiac surgery (left ventricular reconstruction). Out of these, 160 completed all 3 examinations prior to surgical or pathological confirmation of the presence or absence of LVT. DE-CMR for LVT detection in this study had a significantly higher sensitivity and specificity ( $88 \%$ and $99 \%$, respectively) than TTE (23\% and $96 \%$, respectively) or TOE ( $40 \%$ and $96 \%$, respectively) (ref. ${ }^{31}$ ). However, CMR is not suitable as a screening method in all STEMI patients, especially because of its limited availability, long duration and high cost. Thus, echocardiography is the dominant tool in LVT diagnostics.

\section{TREATMENT AND PREVENTION}

In the era of primary PCI, patients after MI usually use DAPT, i.e., acetylsalicylic acid (ASA) and a P2Y receptor inhibitor, for a period of time appropriate to the risk of bleeding, usually for 1 year. Subsequently, ASA therapy is continued for a longer time. There is only a small number of studies on patients after an acute MI complicated by LVT that could determine the optimal length and strategy of antithrombotic therapy to prevent SE while minimizing the risk of bleeding events.

According to the AHA/ACC guidelines, oral vitamin $\mathrm{K}$ antagonists (warfarin) together with DAPT are recom- 
mended for STEMI patients for at least 3 months; possibly longer if there is no increased risk of bleeding and thrombus resolution was incomplete (class IIa recommendation, level of evidence $\mathrm{C}$ ). In addition, preventive administration of warfarin with DAPT (class IIb recommendation, level of evidence $\mathrm{C}$ ) can be considered for anteroapical akinesia or dyskinesia ${ }^{10}$. It should be noted that these guidelines are based on very limited evidence. Based on the recently published European Society of Cardiology (ESC) guidelines from 2017, anticoagulant therapy is indicated for 6 months after the diagnosis of MI and is further adjusted based on the finding of thrombotic residues and the occurrence of bleeding complications (class IIa recommendation, level of evidence C) (ref. ${ }^{9}$ ).

In the study of Maniwa et al., SE occurred in 53\% of cases after 6 months. Therefore the authors recommend anticoagulation for more than 6 months in case of the persistence of the thrombus. In this study, groups of patients with confirmed LVT managed with warfarin together with DAPT (triple therapy) with the time in the therapeutic range (TTR) of $>50 \%$ were compared with a group of patients with TTR $<50 \%$; the effective therapeutic range was defined by somewhat lower INR values 1.6-2.6. Only one embolic event occurred in the TTR $>50 \%$ group, while 9 embolic events were reported in the second group with TTR $<50 \%$, with no significant difference in the incidence of major bleeding events ( $9 \%$ vs. $8 \%$ ). Thus, appropriate anticoagulant therapy with adequate INR values is expected to reduce the risk of embolic events without increasing the incidence of severe bleeding, with a total incidence of severe bleeding events in this study of $1.2 \%$ per year ${ }^{11}$.

More data on the risk of bleeding complications associated with triple therapy have been analysed in patients with AF who have undergone PCI. In a systematic review and meta-analysis, Andrade et al. states that the risk of haemorrhage following PCI was $2.4 \%$ in the first 30 days after the procedure and $4.6 \%$ within 6 months with triple therapy ${ }^{35}$. The incidence of bleeding was 3.3\% in the PIONEER AF-PCI study involving 2,124 patients ${ }^{36}$. In the WOEST study (573 patients), the risk of moderate to severe bleeding (as defined by GUSTO) after 1 year was $12.3 \%$ (ref. ${ }^{37}$ ). However, effective warfarin anticoagulation was defined by therapeutic INR values ranging from 2 to 3 and TTR $>65 \%$ in these studies.

The tactic of the primary preventive antithrombotic strategy is not yet fully understood. In patients with AF, triple therapy has been shown to provide prophylaxis of serious cardiovascular events and reduce all cause mortali$\mathrm{ty}^{38,39}$. This benefit of triple therapy has not been proven in LVT after MI. On the contrary, the risks associated with this medication seem to prevail. In a meta-analysis published in 2017 (including 4 studies), Moulson et al. analysed the effect of warfarin administration in patients with anterior MI, kinetics disorders and reduced LV EF (LV EF about $40 \%$ ). When comparing triple therapy groups of patients (347 patients) to patients with usual therapy after MI, i.e. DAPT (526 patients), there was no significant difference in stroke incidence or mortality, while the incidence of severe bleeding was 2.5 times higher in the patient group with triple therapy ${ }^{13}$. Le May et al. investi- gated the effect of prophylactic warfarin administration in patients after anterior MI treated with primary PCI in echocardiographically detected apical akinesia or dyskinesia. The 460 patients were divided into two groups: 131 patients were on warfarin therapy, 329 without warfarin - the vast majority of patients (more than 97\% in both groups) used DAPT; the therapeutic range of INR was 2-3. There was a significantly higher incidence of NACE (net adverse clinical events) in the group of patients using warfarin $-14.7 \%$ vs. $4.6 \%$. NACE was defined as a composite endpoint including mortality, cerebrovascular accident (CVA) (especially haemorrhagic), reinfarction and severe bleeding 180 days after MI. There was a statistically significantly higher mortality of $5.4 \%$ vs. $4.6 \%$, CVA $3.1 \%$ vs. $0.3 \%$ and severe bleeding $8.5 \%$ vs. $1.8 \%$ (ref. ${ }^{13}$ ). Thus, there is no evidence of a benefit in the form of reduced mortality or CVA incidence, but the incidence of severe bleeding may increase. Therefore, the authors of these recent studies do not recommend the prophylactic administration of warfarin in triple therapy (at INR 2-3), even in high-risk patients with anterior STEMI with reduced LV EF and abnormal apical kinetics.

Regarding the type of the anticoagulant drug used, available literature provides data on the better safety profile of DOAC used in the indication of non-valvular AF after PCI as part of the triple therapy in terms of overall reduction in mortality and relative risk of major bleeding $^{40,41}$. The possibility of LVT treatment with DOAC is not mentioned in recent guidelines, as there is not enough evidence to consider DOAC in this indication. The role of reduced doses of DOAC together with DAPT has been studied in particular in AF patients (e.g. RE-LY trial dabigatran 2x110 mg) and has shown non-inferiority to warfarin, along with a reduction in the incidence of major bleeding events and should be also considered as the primary treatment option in combination with antiplatelet therapy ${ }^{42-44}$. Extrapolation of the results of multicentric trials with DOAC (PIONEER AF-PCI, REDUAL-PCI, AUGUSTUS, ENTRUST AF-PCI), which included predominantly patients with non-valvular atrial fibrillation after primary PCI, could guide the treatment or prophylaxis of LVT after MI, until further research in this area is carried out ${ }^{45,46}$. Thus, reduced doses of DOAC may potentially reduce bleeding complications in this indication. It is therefore necessary to carry out other prospective multicentric studies to confirm or disprove this potential benefit of DOAC treatment.

\section{CONCLUSION}

LVT in the modern PCI era continues to be a serious complication of myocardial infarction, predominantly in the anterior wall. Echocardiography is the basic screening method for the detection and stratification of patient groups with the highest risk of LVT formation. Cardiac MRI provides the highest sensitivity in LVT diagnosis, but it is not suitable as a screening method. Combination antithrombotic therapy, with warfarin anticoagulation together with DAPT, is necessary to reduce the risk of SE 
and prevent ischaemic stroke in patients with already diagnosed LVT after acute MI. Prophylactic administration of warfarin in high-risk groups of patients did not result in reduced mortality or morbidity - on the contrary, the risks associated with this treatment outweighed the benefits. The potential benefit of DOAC in reducing the incidence of apparent SE and in particular the reduction of bleeding complications in this indication have not been sufficiently studied. DOAC should not be administered based on the applicable guidelines and further prospective multicentric studies on this matter should be undertaken.

\section{Search strategy and selection criteria}

Our research strategy was aimed at evaluating studies focused on development of the left ventricular thrombus as complication of myocardial infarction, its diagnosis and treatment. Scientific articles from the period 1980 to 2018 were searched using the PubMed and SCOPUS databases. Search terms included: left ventricular thrombus, myocardial infarction, systemic arterial embolism, stroke, therapy, diagnosis. Only English language papers were reviewed.

Acknowledgement: Supported by the start-up grant of institutional support FNOL 87-85, grant IGA LF UP_2019_008, IGA LF UP_2020_006 and project programme of the Ministry of Health of the Czech Republic with registration no. 17-30101A.

Author contributions: SH, MH, JP, JL, RN, MSp, MS1, DS, ZT, KN, LP, MT: manuscript conception, literature search; SH, JP, MH, DS: manuscript writing; MH, DS, JP, SH, LP, MT: critical revision of manuscript for important intellectual content; SH, JP, DS, ZT, MH, MT: final approval of the submitted manuscript.

All figures are from the authors' archive.

Conflicts of interest statement: None declared

\section{REFERENCES}

1. Zhang Q, Zhang RY, Qiu JP, Zhang JF, Wang $X L$, Jiang $L$, Liao $M L$, Zhang JS, Hu J, Yang ZK, Shen WF. One-year clinical outcome of interventionalist versus patient-transfer strategies for primary percutaneous coronary intervention in patients with acute ST-segment elevation myocardial infarction: results from the REVERSE-STEMI study. Circ Cardiovasc Qual Outcomes 2011;4:355-62.

2. Zhang Q, Zhang RY, Zhang JS, Hu J, Yang ZK, Zheng AF, Zhang X, Shen WF. Outcomes of primary percutaneous coronary intervention for acute ST-elevation myocardial infarction in patients aged over 75 years. Chin Med J (Engl) 2006;119:1151-6.

3. Redfors B, Dworeck C, Angerås O, Haraldsson I, Petursson P, Odenstedt J, loanes D, Völz S, Hiller M, Fransson P, Stewart J, Fryklund $\mathrm{H}$, Albertsson P, Råmunddal T, Omerovic E. Prognosis is similar for patients who undergo primary $\mathrm{PCl}$ during regular-hours and off-hours: A report from SCAAR. Catheter Cardiovasc Interv 2018;91:1240-9.

4. Satler L. Improving outcomes for primary PCl. Cardiovasc Interv 2007;69:497-9.

5. You J, Wang X, Wu J, Gao L, Wang X, Du P, Liu H, Li J, Wang Y, Liang Y, Guo W, Zhang Q. Predictors and prognosis of left ventricular thrombus in post- myocardial infarction patients with left ventricular dysfunction after percutaneous coronary intervention. J Thorac Dis 2018;10:4912-22.

6. Delewi R, Zijlstra F, Piek JJ. Left ventricular thrombus formation after acute myo- cardial infarction. Heart 2012;98:1743-9.
7. Osherov AB, Borovik-Raz M, Aronson D, Agmon $Y$, Kapeliovich M, Kerner A, Grenadier E, Hammerman H, Nikolsky E, Roguin A. Incidence of early left ventricular thrombus after acute anterior wall myocardial infarction in the primary coronary intervention era. Am Heart J 2009;157:1074-80.

8. Weinsaft JW, Kim J, Medicherla CB, Ma CL, Codella NC, Kukar N, Alaref S, Kim RJ, Devereux RB. Echocardiographic algorithm for post-myocardial infarction left ventricular thrombus: a gatekeeper for thrombus evaluation by delayed enhancement CMR. JACC Cardiovasc Imaging 2016;9:505-15.

9. Ibanez B, James S, Agewall S, Antunes MJ, Bucciarelli-Ducci C, Bueno $\mathrm{H}$, Caforio ALP, Crea F, Goudevenos JA, Halvorsen S, Hindricks G, Kastrati A, Lenzen MJ, Prescott E, Roffi M, Valgimigli M, Varenhorst C, Vranckx P, Widimský P; ESC Scientific Document Group. 2017 ESC Guidelines for the management of acute myocardial infarction in patients presenting with ST-segment elevation. The Task Force for the management of acute myocardial infarction in patients presenting with ST-segment elevation of the European Society of Cardiology (ESC). Eur Heart J 2018;39:119-77.

10. O'Gara PT, Kushner FG, Ascheim DD, Casey DE Jr, Chung MK, de Lemos JA, Ettinger SM, Fang JC, Fesmire FM, Franklin BA, Granger CB, Krumholz HM, Linderbaum JA, Morrow DA, Newby LK, Ornato JP, Ou N, Radford MJ, Tamis-Holland JE, Tommaso CL, Tracy CM, Woo YJ, Zhao DX. 2013 ACCF/AHA guideline for the management of STelevation myocardial infarction: a report of the American College of Cardiology Foundation/American Heart Association Task Force on Practice Guidelines. J Am Coll Cardiol 2013;61:e78-e140.

11. Maniwa N, Fujino M, Nakai M, Nishimura K, Miyamoto Y, Kataoka Y, Asaumi Y, Tahara Y, Nakanishi M, Anzai T, Kusano K, Akasaka T, Goto Y, Noguchi T, Yasuda S. Anticoagulation combined with antiplatelet therapy in patients with left ventricular thrombus after first acute myocardial infarction. Eur Heart J 2018;39:201-8.

12. Le May MR, Acharya S, Wells GA, Burwash I, Chong AY, So DY, Glover CA, Froeschl MPV, Hibbert B, Marquis JF, Dick A, Blondeau M, Bernick $J$, Labinaz M. Prophylactic warfarin therapy after primary percutaneous coronary intervention for anterior ST-segment elevation myocardial infarction. JACC Cardiovasc Interv 2015;8:155-62.

13. Moulson N, LaHaye SA, Bertrand OF, MacHaalany J. Prophylactic warfarin post anterior ST-elevation myocardial infarction: A systematic review and meta-analysis. Cardiovasc Revasc Med 2017;18:559-64.

14. Asinger RW, Mikell FL, Elsperger J, Hodges M. Incidence of left- ventricular thrombosis after acute transmural myocardial infarction. Serial evaluation by two-dimensional echocardiography. N Engl J Med 1981;305:297-302.

15. Lamas GA, Vaughan DE, Pfeffer MA. Left ventricular thrombus formation after first anterior wall acute myocardial infarction. Am J Cardiol 1988;62:31-5.

16. Shacham Y, Leshem-Rubinow E, Ben Assa E, Rogowski O, Topilsky Y, Roth A, Steinvil A. Frequency and correlates of early left ventricular thrombus formation following anterior wall acute myocardial infarction treated with primary percutaneous coronary intervention. Am J Cardiol 2013;111:667-70.

17. Solheim S, Seljeflot I, Lunde K, Bjørnerheim R, Aakhus S, Forfang K, Arnesen $\mathrm{H}$. Frequency of left ventricular thrombus in patients with anterior wall acute myocardial infarction treated with percutaneous coronary intervention and dual antiplatelet therapy. Am J Cardiol 2010;106:1197-200.

18. Mao TF, Bajwa A, Muskula P, Coggins TR, Kennedy K, Magalski A, Skolnick DG, Main ML. Incidence of Left Ventricular Thrombus in Patients With Acute ST-Segment Elevation Myocardial Infarction Treated with Percutaneous Coronary Intervention. Am J Cardiol 2018; 121:27-31.

19. Robinson AA, Jain A, Gentry M, McNamara RL. Left ventricular thrombi after STEMI in the primary PCl era: A systematic review and metaanalysis. Int J Cardiol 2016;221:554-9.

20. Delewi R, Nijveldt R, Hirsch A, Marcu CB, Robbers L, Hassell ME, de Bruin RH, Vleugels J, van der Laan AM, Bouma BJ, Tio RA, Tijssen JG, van Rossum AC, Zijlstra F, Piek JJ. Left ventricular thrombus formation after acute myocardial infarction as assessed by cardiovascular magnetic resonance imaging. Eur J Radiol 2012;81:3900-4.

21. Cusick DA, Bonow RO, Chaudhry FA. Left ventricular apical thrombus and myocardial viability: a dobutamine stress echocardiographic study. Echocardiography 2000;17:547-54.

22. Keren A, Goldberg S, Gottlieb S, Klein J, Schuger C, Medina A, Tzivoni 
D, Stern S. Natural history of left ventricular thrombi: their appearance and resolution in the posthospitalization period of acute myocardial infarction. J Am Coll Cardiol 1990;15:790-800.

23. Meltzer RS, Visser CA, Fuster V. Intracardiac thrombi and systemic embolization. Ann Intern Med 1986;104:689-98.

24. Vaitkus PT, Barnathan ES. Embolic potential, prevention and management of mural thrombus complicating anterior myocardial infarction: a meta- analysis. J Am Coll Cardiol 1993;22:1004-9.

25. Barkhausen J, Hunold P, Eggebrecht H, Schüler WO, Sabin GV, Erbe $\mathrm{R}$, Debatin JF. Detection and characterization of intracardiac thrombi on MRI. Am J Roent 2002;179:1539-44.

26. Turhan S, Özcann ÖU, Erol C. Imaging of intracardiac thrombus. Cor Vasa 2013;55:176-83.

27. Cioffi G, Pozzoli M, Forni G, Franchini M, Opasich C, Cobelli F, Tavazzi L. Systemic thromboembolism in chronic heart failure. A prospective study in 406 patients. Eur H J 1996;17:1381-9.

28. Baker DW, Wright RF. Management of heart failure. IV: anticoagulation for patients with heart failure due to left ventricular systolic dysfunction. JAMA 1994;272:1614-8.

29. Lip GYH, Gibbs CR. Antiplatelet agents versus control or antigoagulation for heart failure in sinus rhythm: a Cochrane systematic review. Quarterly Journal of Medicine 2002;95:461-8.

30. Stratton JR, Lighty GW Jr, Pearlman AS, Ritchie JL. Detection of left ventricular thrombus by two-dimensional echocardiography: sensitivity, specificity, and causes of uncertainty. Circulation 1982;66:15666.

31. Kim J, Rodriguez-Diego $S$, Srinivasan A, Brown RM, Pollie MP, Di Franco A, Goldburg SR, Siden JY, Ratcliffe MB, Levine RA, Devereux RB, Weinsaft JW. Echocardiography-quantified myocardial strain a marker of global and regional infarct size that stratifies likelihood of left ventricular thrombus. Echocardiography 2017;34:1623-32.

32. Puskas F, Cleveland JC Jr, Singh R, Weitzel NS, Reece TB, Shull R, Salcedo EE, Seres T. Detection of left ventricular apical thrombus with three-dimensional transesophageal echocardiography. Semin Cardiothorac Vasc Anesth. 2011;15:102-4.

33. Srichai $M B$, Junor $C$, Rodriguez LL, Stillman $A E$, Grimm RA, Lieber ML, Weaver JA, Smedira NG, White RD. Clinical, imaging, and pathological characteristics of left ventricular thrombus: a comparison of contrast-enhanced magnetic resonance imaging, transthoracic echocardiography, and transesophageal echocardiography with surgical or pathological validation. Am Heart J. 2006;152:75-84.

34. Pöss J, Desch S, Eitel C, de Waha S, Thiele H, Eitel I. Left Ventricular Thrombus Formation After ST-Segment-Elevation Myocardia Infarction: Insights From a Cardiac Magnetic Resonance Multicenter Study. Circ Cardiovasc Imaging. 2015;8:e003417.

35. Andrade JG, Deyell MW, Khoo C, Lee M, Humphries K, Cairns JA. Risk of bleeding on triple antithrombotic therapy after percutaneous coronary intervention/stenting: a systematic review and metaanalysis. Can J Cardiol 2013;29:204-12.

36. Gibson CM, Mehran R, Bode C, Halperin J, Verheugt FW, Wildgoose $P$, Birmingham M, lanus J, Burton $P$, van Eickels $M$, Korjian S, Daaboul Y, Lip GY, Cohen M, Husted S, Peterson ED, Fox KA. Prevention of bleeding in patients with atrial fibrillation undergoing $\mathrm{PCl}$. N Engl J Med 2016;375:2423-34.
37. Dewilde WJ, Oirbans T, Verheugt FW, Kelder JC, De Smet BJ, Herrman JP, Adriaenssens T, Vrolix M, Heestermans AA, Vis MM, Tijsen JG, van 't Hof AW, ten Berg JM; WOEST study investigators. Use of clopidogrel with or without aspirin in patients taking oral anticoagulant therapy and undergoing percutaneous coronary intervention: an open-label, randomised, controlled trial. Lancet 2013;381:1107-15.

38. Zhao HJ, Zheng ZT, Wang ZH, Li SH, Zhang Y, Zhong M, Zhang W. "Triple therapy" rather than "triple threat": a meta-analysis of the two antithrombotic regimens after stent implantation in patients receiving long-term oral anticoagulant treatment. Chest 2011;139:260-70.

39. ACTIVE Writing Group of the ACTIVE Investigators, Connolly $S$, Pogue J, Hart R, Pfeffer M, Hohnloser S, Chrolavicius S, Pfeffer M, Hohnloser S, Yusuf S. Clopidogrel plus aspirin versus oral anticoagulation for atrial fibrillation in the Atrial fibrillation Clopidogrel Trial with Irbesartan for prevention of Vascular Events (ACTIVE W): a randomised controlled trial. Lancet 2006;367:1903-12.

40. Chai-Adisaksopha C, Hillis C, Isayama T, Lim W, lorio A, Crowther M. Mortality outcomes in patients receiving direct oral anticoagulants: a systematic review and meta-analysis of randomized controlled trials. J Thromb Haemost 2015;13:2012-20.

41. Ruff $C T$, Giugliano RP, Braunwald E, Hoffman EB, Deenadayalu N, Ezekowitz MD, Camm AJ, Weitz Jl, Lewis BS, Parkhomenko A, Yamashita T, Antman EM. Comparison of the efficacy and safety of new oral anticoagulants with warfarin in patients with atrial fibrillation: a meta-analysis of randomised trials. Lancet 2014;383:955-62.

42. Connolly SJ, Ezekowitz MD, Yusuf S, Eikelboom J, Oldgren J, Parekh A, Pogue J, Reilly PA, Themeles E, Varrone J, Wang S, Alings M, Xavier D, Zhu J, Diaz R, Lewis BS, Darius H, Diener HC, Joyner CD, Wallentin L; RE-LY Steering Committee and Investigators. Dabigatran versus warfarin in patients with atrial fibrillation. N Engl J Med 2009;361:113951.

43. Dans AL, Connolly SJ, Wallentin L, Yang S, Nakamya J, Brueckmann M, Ezekowitz M, Oldgren J, Eikelboom JW, Reilly PA, Yusuf S. Concomitant use of antiplatelet therapy with dabigatran or warfarin in the Randomized Evaluation of Long-term Anticoagulation Therapy (RE-LY) trial. Circulation 2013;127:634-40.

44. Angiolillo DJ, Goodman SG, Bhatt DL, Eikelboom JW, Price MJ, Moliterno DJ, Cannon CP, Tanguay JF, Granger CB, Mauri L, Holmes DR, Gibson CM, Faxon DP. Antithrombotic Therapy in Patients With Atrial Fibrillation Undergoing Percutaneous Coronary Intervention: A North American Perspective-2016 Update. Circ Cardiovasc Interv 2016;9:e004395.

45. Cannon CP, Bhatt DL, Oldgren J, Lip GYH, Ellis SG, Kimura T, Maeng M, Merkely B, Zeymer U, Gropper S, Nordaby M, Kleine E, Harper R, Manassie J, Januzzi JL, Ten Berg JM, Steg PG, Hohnloser SH; RE-DUAL $\mathrm{PCl}$ Steering Committee and Investigators. Dual Antithrombotic Therapy with Dabigatran after $\mathrm{PCl}$ in Atrial Fibrillation (RE-DUAL PCI). N Engl J Med 2017;377:1513-24.

46. Gibson CM, Mehran R, Bode C, Halperin J, Verheugt FW, Wildgoose $P$, Birmingham $M$, lanus J, Burton $P$, van Eickels $M$, Korjian S, Daaboul Y, Lip GY, Cohen M, Husted S, Peterson ED, Fox KA. Prevention of bleeding in patients with atrial fibrillation undergoing $\mathrm{PCl}$. N Engl J Med 2016;375:2423-34. 